\title{
Reflexões sobre o uso do big data em modelos preditivos de vigilância epidemiológica no Brasil
}

Reflections on the use of big data in predictive models for epidemiological surveillance in Brazil

Reflexiones sobre el uso de macrodatos en modelos predictivos de vigilancia epidemiológica en Brasil

Rui Massato Harayama ${ }^{1}$

\section{Resumo}

Objetivo: discutir as implicações bioéticas a partir do anúncio da Saúde Digital por parte da Organização Mundial da Saúde e do uso do big data na produção de sistemas preditivos de vigilância em saúde no Brasil. Metodologia: realizou-se revisão narrativa a partir da busca de artigos nas plataformas Scielo, Bireme, Jstor e na página da Organização Mundial da Saúde e do Ministério da Saúde do Brasil, com os descritores big data, bioética e ética, de maio a julho de 2020. Resultados: foram evidenciados limites no uso do big data como ferramenta de vigilância epidemiológica preditiva, notadamente com o seu uso durante a pandemia de Covid-19, apesar de justificável a partir da teoria da bioética da proteção e da ética da saúde pública. Os maiores limites observados foram ausência de legislação de proteção de dados adequada e viés dos dados obtidos. Conclusão: para análise dos impactos bioéticos do uso do big data na medicina do futuro é imprescindível aprofundar a discussão sobre os possíveis impactos que o uso dessas tecnologias pode gerar na vida em sociedade, com ênfase no desenvolvimento do capitalismo de vigilância, na interferência na vida social e no acirramento das desigualdades regionais.

Palavras-chave: Bioética. Big data. Vigilância em saúde pública. Ética.

\begin{abstract}
Objective: to discuss the bioethical implications from the announcement of Digital Health by the World Health Organization and the use of big data in the production of predictive health surveillance systems in Brazil. Methods: conducted a narrative review based on the search for articles on Scielo, Bireme, Jstor platforms and at the World Health Organization and the Brazilian Ministry of Health webpages, with the keywords big data, bioethics and ethics, from May to July 2020. Results: limits were evidenced in the use of big data as an epidemiological forecasting surveillance tool, notably with its use during the Covid-19 pandemic, although justified by the Bioethics of Protection Theory and Public Health Ethics. The greatest limits observed were the absence of adequate data protection legislation and bias in the data obtained. Conclusion: in order to analyze the bioethical impacts of the use of big data in the medicine of the future, it is essential to deepen the discussion on the possible impacts that the use of these technologies can have on life in society, with an emphasis on the development of surveillance capitalism, on interference in life and the intensification of regional inequalities.
\end{abstract}

Keywords: Bioethics. Big data. Public health surveillance. Ethics.

\footnotetext{
${ }^{1}$ Mestre em Antropologia Social; professor, Instituto de Saúde Coletiva, Universidade Federal do Oeste do Pará, Santarém, Pará, Brasil. https://orcid.org/0000-0002-7231-3589. E-mail: rui.harayama@gmail.com
} 


\section{Resumen}

Objetivo: discutir las implicaciones bioéticas del anuncio de la Salud Digital por la Organización Mundial de la Salud y el uso de macrodatos en la producción de sistemas de vigilancia predictiva de salud en Brasil. Metodología: se realizó una revisión narrativa a partir de la búsqueda de artículos en las plataformas Scielo, Bireme, Jstor y en la página de la Organización Mundial de la Salud y el Ministerio de Salud de Brasil, con las palabras clave macrodatos, bioética y ética, de mayo a julio, 2020. Resultados: se evidenciaron límites en el uso de macrodatos como herramienta de vigilancia epidemiológica predictiva, destacando su uso durante la pandemia Covid-19, aunque justificado a partir de la teoría de la Bioética de Protección y la Ética en Salud Pública. Los mayores límites observados fueron la ausencia de una legislación de protección de datos adecuada y el sesgo en los datos obtenidos. Conclusión: para analizar los impactos bioéticos del uso de macrodatos en la medicina del futuro, es fundamental profundizar la discusión sobre los posibles impactos que el uso de estas tecnologías puede tener en la vida en sociedad, con énfasis en el desarrollo del capitalismo de vigilancia, en la interferencia en la vida y la intensificación de las desigualdades regionales.

Palabras clave: Bioética. Macrodatos. Vigilancia de salud pública. Principio moral.

\section{Introdução}

O desenvolvimento da tecnologia digital e sua interface na área da saúde tem trazido implicações bioéticas para os profissionais de saúde e sistemas de saúde. Philippe Meyer, em sua obra $A$ irresponsabilidade médica (1), previa que a medicina do século XXI seria extremamente tecnicista, deixando de cuidar do ser humano em sua integralidade e passando a cuidar de organelas celulares. Para o autor, quando a medicina passasse seus cuidados aos níveis celulares, o impacto moral da decisão das terapias preditivas, como as genômicas, ou da fertilização in vitro, seria deixado a cargo de bioeticistas, formados em centros de filosofia, e que estariam organizados em comitês de bioética nacionais. $\mathrm{O}$ autor questiona os impactos da delegação da reflexão da bioética entre especialistas que desobrigaria médicos de refletirem as implicações éticas dos seus atos (1).

Esse descomprometimento moral do resultado das intervenções de saúde por parte dos profissionais de saúde é o que o autor definiu como sendo a irresponsabilidade médica. Essa irresponsabilidade seria o resultado de uma extrema fragmentação dos procedimentos e das especializações dentro dos sistemas de saúde (1).

Nesse contexto, o objetivo deste artigo é discutir as implicações bioéticas do uso das tecnologias digitais (2), a partir do anúncio da saúde digital por parte da Organização Mundial da Saúde (OMS) e do uso do big data na produção de sistemas preditivos de vigilância em saúde no Brasil (3). 


\section{Metodologia}

Este estudo é uma revisão narrativa sobre big data e suas implicações bioéticas. Como aponta Rother (4), revisões narrativas são amplas e apropriadas para descrever o estado de arte de determinado assunto, a partir de um ponto de vista contextual.

Para o objetivo do artigo, a metodologia justifica-se pelo crescente interesse da temática presente em diferentes áreas do conhecimento. Os textos analisados foram pesquisados de forma não sistemática nos meses de maio a julho de 2020 nas plataformas Scielo, Bireme, Jstor e em sites oficiais como da OMS e do Ministério da Saúde (MS), a partir dos descritores big data, bioética e ética. Como critério de inclusão, foram adotados artigos disponíveis online, publicados nos últimos cinco anos em português, inglês, espanhol ou francês. Após leitura do resumo, foram excluídos os artigos que não discorriam sobre o uso de modelos matemáticos preditivos, tecnologias digitais e vigilância em saúde. Ao total, foram utilizados 21 artigos.

\section{O surgimento da saúde digital como questão global}

Os teóricos da informação relatam que desde o surgimento da internet e a criação da world wide web (5), em 1989, a rede evolui e transformou-se em um enorme repositório de produção e compartilhamento de conhecimento com uso intenso da inteligência artificial na fase da web 3.0, também chamada de internet semântica (6). As transformações da internet e seu desenvolvimento tecnológico culminariam na próxima revolução industrial: a indústria 4.0 .

De forma resumida, a indústria 4.0 é aquela na qual há uma interação profunda entre seres humanos, máquina, inteligência artificial e algoritmos (7). O potencial dessa nova revolução industrial acarretaria no surgimento de uma saúde 4.0 (8), caracterizada pela aceleração da interoperabilidade de grandes bancos de dados e permitindo que os sistemas de saúde se tornem cada vez mais personalizados, eficientes, preditivos e baratos. Como exemplo, podemos citar o desenvolvimento de impressoras 3D, que diminuíram os custos na produção de equipamentos tecnológicos, próteses e insumos projetados especificamente para o paciente e produzidos de acordo com a demanda. Ou ainda na socialização de prontuários de pacientes, que poderiam receber o melhor tratamento para suas patologias, incluindo o atendimento via telessaúde, realizado por qualquer profissional do mundo ou em localidades remotas (9). Do ponto de vista preventivo, a alimentação constante de um 
prontuário eletrônico poderia prever o futuro clínico de um paciente apenas correlacionando o histórico de saúde de pessoas com doenças como câncer, diabetes, Alzheimer, entre outros exemplos de usos da saúde $4.0(10,11)$.

Do ponto de vista da saúde global, o interesse da OMS com as interfaces tecnológicas não é recente: desde de 2005 a instituição vem propondo ações aos estados signatários no sentido de implementar uma interface digital aos seus sistemas de saúde (5).

Entretanto, o tema da saúde digital consolida-se em 2018 na 71를 Assembleia Mundial da Saúde, quando foi aprovada a recomendação WHA 71.7 sobre a saúde digital. Essa recomendação reconhece a importância das tecnologias digitais como forma de promover a saúde e prevenir doenças, aumentando a acessibilidade e qualidade dos serviços de saúde (5). A recomendação WHA 71.7 também reforça os Objetivos do Desenvolvimento Sustentável, que propõem a cooperação entre países na transferência de tecnologias, garantindo o acesso à tecnologia para todos até 2030.

Aos estados-membros, a recomendação aponta 11 tópicos, entre eles 0 fortalecimento e integração dos sistemas nacionais de informação em saúde e a capacitação de profissionais de saúde. Para a presente análise, o destaque deve ser dado a dois tópicos: i) o item 10, que indica a necessidade do desenvolvimento de legislação específica para a proteção e segurança de dados em consonância com as garantias internacionais de direitos humanos e; ii) o item 11, que propõe o desenvolvimento do uso da saúde digital em colaboração com diversos setores (5).

A partir dessa síntese, observa-se que a recomendação WHA 71.7 tem um caráter intersetorial em dois pontos sensíveis: a interface com a legislação e com a indústria da tecnologia.

Com o objetivo de implementar a recomendação da saúde digital, a OMS institui, em outubro de 2019, um departamento técnico de saúde digital e um grupo técnico consultivo de especialistas que propuseram a Estratégia Global para Saúde Digital (2020-2024) (17). Nesse documento, consolida-se o termo de saúde digital como sendo um guarda-chuva, que seria o campo de conhecimento e prática associados ao desenvolvimento e uso de tecnologias digitais para melhorar a saúde. A saúde digital expande o conceito de eSaúde para incluir consumidores digitais, com uma gama mais ampla de dispositivos inteligentes e equipamentos conectados. Também abrange outros usos das tecnologias digitais para a saúde, como a internet das coisas, inteligência artificial, big data e robótica (11). 
Com essa conceituação oficial, observa-se a pluralidade de significados e tecnologias que o termo saúde digital inclui, sendo necessário um recorte nos possíveis usos do big data na saúde e as implicações bioéticas que essa tecnologia apresenta.

\section{O big data na saúde}

O termo big data refere-se ao uso de dados complexos e em grandes quantidades definidos por quatro dimensões: volume, velocidade, variedade e veracidade (10). O crescimento acelerado de bancos de dados com informações da saúde tem colocado o uso do big data como uma ferramenta para análise e predição de doenças. Epidemiologistas que têm trabalhado com big data defendem o seu uso como instrumento preditivo para antecipar surtos e epidemias de doenças (11).

Uma das potencialidades permitidas com a intercambialidade entre bancos de informações e dados está no cruzamento de diferentes bases, como a de prontuário de saúde, dados de geolocalização, perfil de uso de redes sociais, dados de pagamentos bancários, entre outros (12). Como pode ser observado, o big data não utiliza somente dados relativos à saúde do indivíduo, que pode dar anuência para o uso de seus dados pessoais, mas a busca propositiva, por meio de algoritmos e de inteligência artificial, correlacionando com outras bases de dados, por padrões e informações que possam auxiliar na compreensão da saúde de determinada população ou indivíduo (13).

Ou seja, para conseguir que o big data forneça esse tipo de predição, epidemiologistas apostam nos bancos de dados gerados por meio da internet da coisas no qual diferentes dispositivos enviam informações dentro de nuvens digitais que podem ser acessadas, consultadas e comparadas (11). Conforme as orientações da OMS, esses sistemas devem ser construídos a partir de legislações que garantam a segurança digital desses sistemas e o uso ético dos dados, sobretudo dados pessoais e sigilosos que podem infringir a privacidade dos cidadãos (14).

No Brasil, o caráter universal da assistência à saúde preconizada na Constituição de 1988 (15) fomentou o desenvolvimento de um sistema nacional de informação. No contexto do Sistema Único de Saúde (SUS), o DataSUS (16) armazena e disponibiliza dados de saúde sem identificação pessoal para a população, os gestores e os pesquisadores, com o objetivo de consulta, análise e planejamento. Uma das características que diferenciam o DataSus, em sua versão atual, do big data, está na ausência de interoperabilidade e 
rastreabilidade da fonte de informação. Ou seja, o DataSus com uso em interface com o big data possibilitaria, a partir do banco de dados, chegar ao indivíduo que possui determinada patologia, cujo estilo de vida aponta para um risco a determinadas doenças ou, ainda, que circulou por espaços considerados inadequados ou insalubres. Coloca-se, aqui, questões éticas e legais sobre o risco de mau uso das informações armazenados ou da exposição de dados pessoais e sigilosos.

\section{A garantia da privacidade dos dados no Brasil}

Com o objetivo de evitar possíveis infrações éticas decorrentes do mau uso do big data, faz-se necessário a implementação de um arcabouço legal de proteção de dados, como orienta a OMS (17). No Brasil, em 2018, foi promulgada a Lei Geral de Proteção dos Dados (18), que garante o sigilo dos dados pessoais e resguarda os cidadãos dos maus usos dos dados por parte dos governos. Essa lei prevê o uso de dados pessoais em questões de segurança nacional ou sanitária, colocando esse instrumento legal, do ponto de vista das garantias das liberdades, dentro de um paradoxo que precisa ser observado e discutido (19).

Para ilustrar esse paradoxo, podemos realizar um paralelo com a Lei de Acesso à Informação, de 2011 (20), que previa a publicização ativa dos dados produzidos por órgãos públicos, incluídos os processos judiciais. Apesar do sigilo da condição de diagnóstico ser garantida por lei (21), uma mineração de dados, estratégia do big data em saúde, conseguiria cruzar diferentes bancos de dados, listar nominalmente portadores de doenças raras que judicializaram o seu acesso a tratamentos e comparar o custo anual dessa decisão aos custos do tratamento de uma doença crônica não-transmissível.

Mesmo sem a necessidade de medida judicial, já é possível realizar o cruzamento de perfis públicos com temas de interesse da saúde pública, como suicídio entre jovens (22), sendo aventado como ferramenta de vigilância epidemiológica. Nesse tipo de uso, busca-se encontrar usuários com determinadas tendências, a partir da análise das suas postagens em redes sociais, tornando-se em ferramenta de vigilância em saúde preditiva (23). Esses exemplos ilustram os riscos legais e éticos para a autonomia e privacidade dos indivíduos decorrentes do uso do big data como ferramenta de vigilância em saúde preditiva, sobretudo em temas de segurança nacional ou de saúde pública. 
Com o advento da pandemia da Sars-Cov-2, uma das estratégias utilizadas por países na contenção do vírus foi o desenvolvimento de algoritmos e indicadores a partir dos dados digitais produzidos por aparelhos celulares ou aplicativos de redes sociais. Como aponta as recomendações da OMS (14), o tipo de resposta produzida em contexto de pandemia, em seu caráter excepcional, pode trazer prejuízos às liberdades individuais e infrações aos direitos humanos, caso não sejam implementados com segurança legal. Apesar da possibilidade da coleta de dados ser feita com protocolos de criptografia, o caráter inovador e dinâmico da ciência da informação muitas das vezes colocam à disposição do governo e da população, de forma gratuita, aplicativos de empresas privadas ou startups de tecnologia. Essas empresas podem disponibilizar aplicativos gratuitos em troca do uso comercial dos dados coletados para outros fins que os definidos no momento da contratação dos serviços. Tal prática coloca em discussão a real segurança dos dados e, sobretudo, o limite da autonomia dos indivíduos nessas situações em que governos cedem a prerrogativa do uso de dados pessoais a empresas particulares.

Com a intensificação do uso desses aplicativos digitais, a OMS emitiu recomendação específica sobre as questões éticas envolvendo o uso de aplicativos de proximidade, alertando para a ausência de evidências científicas que indiquem o seu uso. Também reforçou os riscos que envolvem o uso de aplicativos corporativos de empresas privadas, com relação ao armazenamento desses dados (14).

Com indicação semelhante, Bioni et al (24) recomendam que o uso de dados digitais, no contexto de pandemias como a Covid-19, obedeça 10 princípios, entre eles o de motivação, ciclo de vida, anonimização e segurança da informação. Para os autores, o uso de dados digitais para fins de saúde pública só deve ocorrer com motivação clara, por meio de aplicativos de código aberto e que após o fim do ciclo de uso sejam descartados (24).

lenca e Vayena (25) apresentam uma matriz semelhante ao analisar o uso de dados digitais produzidos por aparelhos celulares como instrumento de combate à Covid-19. Para os autores três princípios éticos deveriam ser observados: i) a proporcionalidade para a seriedade do risco à saúde pública; ii) limitar-se ao necessário para atingir o objetivo de saúde pública e; iii) ser cientificamente justificado (25).

Os autores demonstram que a suspensão dos direitos individuais sobre os dados digitais justifica-se proporcionalmente ao risco de saúde pública (25), ou seja, não seria uma suspensão total da autonomia dos indivíduos, mas no que tange aos perigos colocados à 
coletividade, como na exigência do isolamento individual de pessoas infectadas. Alertam que os usos da tecnologia devem ser informados constantemente à população para garantir adesão e confiança da população no uso dos dados digitais.

As orientações éticas para o uso do big data no contexto da pandemia reforçam a necessidade da temporalidade do armazenamento dos dados, preferencialmente em aplicativos governamentais $(14,17,24)$. O outro eixo dessas recomendações centra-se em uma análise que pondera direitos individuais e direitos coletivos em relação à privacidade e autonomia. Nesse sentido, parece-nos que a análise ética do uso do big data na saúde pode ser analisada a partir da bioética da proteção, que se debruça sobre a reflexão de uma bioética sanitária. A bioética da proteção tem seu quadro teórico e analítico voltado para a implementação de políticas de saúde e proteção das coletividades (26). Do ponto de vista da bioética da proteção, o uso consensuado de informações pessoais pode ser justificado em favor de um bem maior, como na segurança sanitária de uma população (26). Seguindo argumento similar, Evans (27) defende o senso de coletividade e bem comum como elementos centrais para a análise bioética do uso do big data.

Nesse sentido, concordamos com matriz de Childress (28), que foi retomada por Chung et al (29), ao propor que a análise ética do uso do big data deva estar baseada na ética da saúde pública e analisada a partir dos seguintes tópicos: efetividade, proporcionalidade, necessidade, redução de infrações e justificativa pública.

Como resposta à saúde pública, o uso de big data como instrumento preditivo cumpre os quesitos de justificativa pública, necessidade e proporcionalidade. Entretanto, ainda se faz necessário aprofundar discussões a cada aplicação do big data na saúde acerca da efetividade e da redução de infrações viabilizadas por essa tecnologia.

\section{Apontamentos críticos ao uso do big data}

A partir da exposição das implicações éticas do uso do big data e de como essa tecnologia foi analisada no contexto da pandemia da Covid-19, torna-se fundamental apontar os limites do uso da mesma. Apesar do uso do big data ser recomendado pela OMS como uma ferramenta para a saúde digital e para a medicina do futuro, autores tem tecido críticas quanto ao seu uso como ferramenta efetiva para a vigilância preditiva em saúde.

Bruno et al (12) associam a prática preditiva do uso de algoritmos do big data a um capitalismo de dados. Nessa forma de relação, o padrão de uso das redes digitais estaria 
associado a uma vigilância de dados sem o consentimento dos indivíduos, e o conhecimento gerado pelos algoritmos seria baseado em correlações frágeis (30).

A pandemia da Covid-19 demonstrou alguns limites do big data como instrumento preditivo. A análise de dados, para ser efetivo, depende de banco de dados qualificados (31) e de sistemas de vigilância em saúde ativos (32). Soma-se a isso, o fato de que mesmo em países ou regiões desenvolvidos, o uso de aplicativos digitais ou informações geradas por celulares acabam gerando vieses econômico e etário. Em Cingapura, o cluster de casos ocorreu entre trabalhadores que não tinham condições econômicas para a manutenção de smartphones ou celulares com pacotes de dados (31). Um viés semelhante ocorreria no Brasil, onde 30\% dos municípios não possuem acesso à internet de qualidade (33).

O uso voluntário indicado por órgãos como a OMS (14), quando realizado sem evidência científica, pode gerar dados enviesados ou sem uso prático para as políticas públicas (34) - os dados gerados não teriam finalidade para a vigilância em saúde. Sem uma justificativa adequada para o uso desses dados, incorre-se no perigo de gerar análises estigmatizantes de determinadas áreas ou do público que faz uso de determinados serviços (35) (36).

Também deve ser ressaltado que há crítica na literatura sobre a implicação ética do uso do big data no meio social, a partir da evidência de que os algoritmos têm sido utilizados como formas de controlar comportamentos sociais (30). Há evidências de que o uso dos algoritmos interfere nas bolsas de valores (37), nas eleições (38) e na forma de estratificação das redes de socialização (30).

O uso da big data em saúde como instrumento de predição de doenças não deve se sobrepor às medidas clássicas e efetivas de busca ativa e comunicação em saúde - que implicam a presença constante de equipes de saúde pública nos territórios -, nem se basear somente em demandas internacionais de doenças e agravos à saúde global (39).

\section{Considerações finais}

O crescente interesse pela saúde digital tem trazido implicações para pesquisadores e gestores. Nesse sentido, torna-se fundamental compreender a especificidade das diversas tecnologias que a saúde digital abrange.

Neste artigo, foram trazidas algumas reflexões éticas sobre o uso do big data em saúde dentro do contexto brasileiro. Apesar das possibilidades de uso como ferramenta 
preditiva, observamos que a sua eficácia se torna baixa quando se respeitam as legislações brasileiras de proteção de dados. Em contextos de pandemia, a aplicação do big data em saúde como ferramenta preditiva tem baixa eficácia, com dados enviesados e que exclui estratos econômicos mais baixos e grupos etários, apesar de justificável eticamente a partir da bioética da proteção e da ética da saúde pública.

Ressalta-se que, no contexto brasileiro, o uso do big data em saúde pode acirrar o desiquilíbrio já existente na vigilância em saúde.

\section{Referências}

1. Meyer P. A irresponsabilidade médica, São Paulo: Unesp; 2002. 133p.

2. Langarizadeh $M$, Moghbeli F, Aliabadi A. Application of Ethics for Providing Telemedicine Services and Information Technology. Med Arch. 2017;71(5):351-355. Disponível em: https://doi.org/10.5455/medarh.2017.71.351-355.

3. Wyber R, Vaillancourt S, Perry W, Mannava P, Folaranmi T, Celi LA. Big data in global health: improving health in low- and middle-income countries. Bull World Health Organ. 2015; 93(3):203-208. Disponível em: https://doi.org/10.2471/BLT.14.139022.

4. Rother ET. Revisão sistemática X revisão narrativa. Acta paul. enferm. [Internet]. 2007 June [acesso em 26 de julho de 2020]; 20(2): v-vi. Disponível em:

http://www.scielo.br/scielo.php?script=sci_arttext\&pid=S0103$21002007000200001 \& \operatorname{lng}=$ en.

5. Organização Mundial da Saúde. Digital health resolution. In: Septuagésima Primeira Assembleia Geral da Organização Mundial de Saúde. 26.mai.2018 [Acesso em 15.jun.2020]. Disponível em: http://apps.who.int/gb/ebwha/pdf_files/WHA71/A71_R7-en.pdf.

6. Centro de Estudos sobre Tecnologias Web. Linha do tempo da Web. [s.d.] [Acesso em 15.jun.2020]. Disponível em: https://ceweb.br/linhadotempo/

7. Andrade P. Intelectuais e web 2.0/3.0: como pensar, no $3^{\circ}$ milênio, a utopia do intelectual. In: Sousa CM, org. Um convite à utopia [online]. Coleção Um convite à utopia, vol. 1. Campina Grande: EDUEPB; 2016. p. 365-398. Disponível

em: https://doi.org/10.7476/9788578794880.0011.

8. Arbix G, Salerno MS, Zancul E, Amaral G, \& Lins LM. (2017). O Brasil e a nova onda de manufatura avançada: o que aprender com Alemanha, China e Estados Unidos. Novos estudos CEBRAP, 36(3), 29-49. Disponível em: https://doi.org/10.25091/s01013300201700030003.

9. Lopes MACQ, Oliveira GMM, Maia LM. Saúde digital, direito de todos, dever do Estado? Arq. Bras. Cardiol. [Internet]. 2019 Sep [Acesso em 26.jul.2020]; 113(3): 429-434. 
Disponível em: http://www.scielo.br/scielo.php?script=sci_arttext\&pid=S0066782X2019000900429\&Ing=en.

10. Valentim RAM, Morais AHF, Silva Neto JHV, Guimaraes MCS, Lima TS. Conectividade e Digitalização no Contexto da Saúde Global: um olhar para o futuro inspirado na saúde 4.0. In: Fotini Santos Toscas, Thiago Rodrigues Santos. (Org.). Avanços, Desafios e Oportunidades no Complexo Industrial da Saúde em Serviços e Tecnologias. Brasília: Ministério da Saúde; 2018, v. 1. p. 254-273.

11. Chiavegatto Filho ADP. Uso de big data em saúde no Brasil: perspectivas para um futuro próximo. Epidemiol. Serv. Saúde [Internet].; 24(2): 325-332. Disponível em: https://doi.org/10.5123/S1679-49742015000200015.

12. Bruno FG, Bentes ACF, Faltay P. Economia psíquica dos algoritmos e laboratório de plataforma: mercado, ciência e modulação do comportamento. Revista Famecos. 2019; 26(3), e33095. Disponível em: https://doi.org/10.15448/1980-3729.2019.3.33095.

13. Klein GH, Guidi Neto P, Tezza R. Big Data e mídias sociais: monitoramento das redes como ferramenta de gestão. Saude soc. [Internet]. 2017 Mar; 26(1): 208-217. Disponível em: https://doi.org/10.1590/s0104-12902017164943.

14. Organização Mundial da Saúde. Ethical considerations to guide the use of digital proximity tracking technologies for COVID-19 contact tracing. 28. maio.2020 [Acesso em 15.jun.2020]. Disponível em: https://www.who.int/publications/i/item/WHO-2019-nCoVEthics_Contact_tracing_apps-2020.1.

15. Brasil. Constituição da República Federativa do Brasil. Diário Oficial da União, 5 de outubro de 1988.

16. Ministério da Saúde. DataSus: histórico. [s.d.] [Acesso em 15.jun.2020]. Disponível em: https://datasus.saude.gov.br/sobre-o-datasus/

17. World Health Organization. Global Strategy on Digital Health. 2020-2024 [Acesso em 26.jul.2020]. Disponível em: https://extranet.who.int/dataform/upload/surveys/18343.

18. Brasil. Lei no 13.709, 14 de agosto de 2018. Lei Geral de Proteção de Dados Pessoais (LGPD). 15 ago. 2018 [Acesso em 15.jun.2020]. Disponível em:

http://www.planalto.gov.br/ccivil_03/_ato2015-2018/2018/lei/L13709.htm.

19. Szinvelski MM, Arceno TS, Francisco LB. Perspectivas jurídicas da relação entre big data e proteção de dados. Perspectivas em Ciência da Informação. 2019; 24(4): 32-144. Disponível em: https://doi.org/10.1590/1981-5344/4188.

20. Brasil. Lei n. 12.527, de 18 de novembro de 2011. Regula o acesso a informações previsto no inciso XXXIII do art. 5o, no inciso II do $\S 30$ do art. 37 e no $\S 20$ do art. 216 da Constituição Federal; altera a Lei no 8.112, de 11 de dezembro de 1990; revoga a Lei no 11.111, de 5 de maio de 2005, e dispositivos da Lei no 8.159, de 8 de janeiro de 1991; e dá outras providências. Brasília, DF: Diário Oficial [da] República Federativa do Brasil; 18 nov. 
2011 [Acesso em 2.jan.2013]. Disponível em:

http://www.planalto.gov.br/ccivil_03/_ato2011-2014/2011/lei/l12527.htm.

21. Lütz KT, Carvalho D, Bonamigo EL. Sigilo profissional: conhecimento de alunos de medicina e médicos. Rev. Bioét. [Internet]. 2019 Sep [Acesso em 26.jul.2020]; 27(3): 471481. Disponível em: http://www.scielo.br/scielo.php?script=sci_arttext\&pid=S1983$80422019000300471 \&$ lng=en doi.org/10.1590/1983-80422019273331.

22. Fonseka TM, Bhat V, Kennedy SH. The utility of artificial intelligence in suicide risk prediction and the management of suicidal behaviors. Australian \& New Zealand Journal of Psychiatry. 53(10), 954-964. https://doi.org/10.1177/0004867419864428.

23. Klein Gisiela Hasse, Guidi Neto Pedro, Tezza Rafael. Big Data e mídias sociais: monitoramento das redes como ferramenta de gestão. Saude soc. [Internet]. 2017 Mar; 26(1): 208-217. Disponível em: https://doi.org/10.1590/s0104-12902017164943.

24. Bioni B, Zanatta R, Monteiro R, Rielli M. Privacidade e pandemia: recomendações para o uso legítimo de dados no combate à COVID-19. Conciliando o combate à COVID-19 com o uso legítimo de dados pessoais e o respeito aos direitos fundamentais. São Paulo: Data Privacy Brasil; 2020.

25. lenca M, Vayena E. On the responsible use of digital data to tackle the COVID-19 pandemic. Nat Med. 2020; 26, 463-464. Disponível em: https://doi.org/10.1038/s41591020-0832-5.

26. Schramm FR. A bioética de proteção: uma ferramenta para a avaliação das práticas sanitárias? Ciênc. saúde coletiva [Internet]. 2017 maio [Acesso em 26.jul.2020]; 22(5): 1531-1538. Disponível em: http://dx.doi.org/10.1590/1413-81232017225.04532017.

27. Evans B. Big Data and Individual Autonomy in a Crowd. In: I Cohen, Lynch H, Vayena E, Gasser U (eds.). Big Data, Health Law, and Bioethics. Cambridge: Cambridge University Press; 2018. p. 19-29. Disponível em: https://doi.org/10.1017/9781108147972.003.

28. Childress, JF, Faden RR, Gaare RD, Gostin LO, Kahn J, Bonnie RJ, Kass NE, Mastroianni AC, Moreno JD, Nieburg P. Public Health Ethics: Mapping the Terrain. The Journal of Law, Medicine \& Ethics. 2002; 30: 170-178. Disponível em: https://doi.org/10.1111/j.1748-720X.2002.tb00384.X.

29. Chung RYN, Erler A, Li HL, Au D. Using a Public Health Ethics Framework to Unpick Discrimination in COVID-19 Responses. The American Journal of Bioethics. 2020; 20:7, 114-116. Disponível em: https://doi.org/10.1080/15265161.2020.1779403.

30. Bruno F. Rastrear, classificar, performar. Cienc. Cult. [Internet]. 2016 Mar [acesso em 26 de julho de 2020]; 68(1): 34-38. Disponível em: http://dx.doi.org/10.21800/231766602016000100012 .

31. Robinson K. A false promise of COVID-19 'big' health data? Health data integrity and the ethics and realities of Australia's health information management practice. Health 
Information Management Journal. 2020. Disponível em: https://doi.org/10.1177/1833358320941190.

32. Rizzo E. COVID-19 contact tracing apps: the 'elderly paradox'. Public Health. 2020 Jun 28;185:127. Disponível em: https://doi.org/10.1016/j.puhe.2020.06.045. Epub ahead of print. PMID: 32622218; PMCID: PMC7321037.

33. Anatel. Mapeamento de redes de transporte. 2019 [Acesso em 15.jun.2020]. Disponível em: https://www.anatel.gov.br/dados/mapeamento-de-redes.

34. Mittelstadt B. From Individual to Group Privacy in Biomedical Big Data. In: Cohen I, Lynch H, Vayena E, Gasser U (eds.). Big Data, Health Law, and Bioethics (). Cambridge: Cambridge University Press; 2018. p. 175-192. Disponível em: https://doi.org $10.1017 / 9781108147972.017$

35. Wahlert L. Mapping Queer Bioethics: Space, Place, and Locality. Journal of Homosexuality. 2016; 63:3. p. 301-305. Disponível em: https://doi.org/10.1080/00918369.2016.1124686.

36. Associated Press. Gay dating app Grindr shared HIV status with other companies. Taiwan News. 03 abr 2018 [Acesso em 15.jun.2020]. Disponível em: https://www.taiwannews.com.tw/en/news/3397423.

37. Paraná E. A digitalização do mercado de capitais no Brasil: tendências recentes. Boletim de Economia e Política Internacional. 2017; n. 23. p. 43-71.

38. Caldas CLM, Caldas PNL. Estado, democracia e tecnologia: conflitos políticos e vulnerabilidade no contexto do big-data, das fake news e das shitstorms. Perspect. ciênc. inf. [online]. 2019; vol. 24 (2): 196-220. Disponível em: https://doi.org/10.1590/1981$5344 / 3604$.

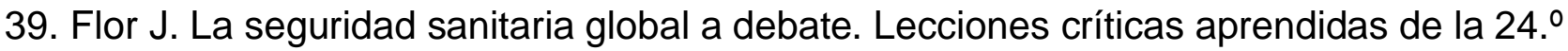
EVE. Comillas Journal of International Relations. 2018. p. 49-62. Disponível em: https://doi.org/10.14422/cir.i13.y2018.004. 